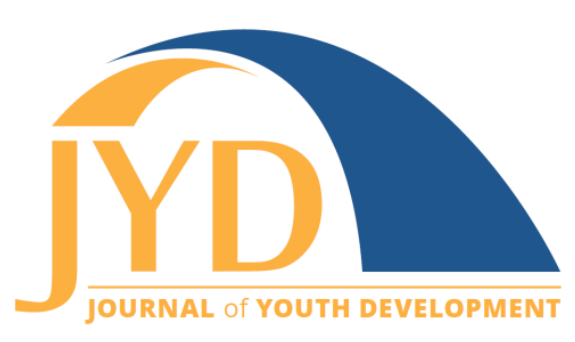

http://jyd.pitt.edu/ | Vol. 15 Issue 6 DOI 10.5195/jyd.2020.938 | ISSN 2325-4017 (online)

\title{
Latinx Adolescents' Peer Ethnic Discrimination in After-School Activities and Activity Experiences
}

\author{
Ting-Lan Ma \\ School of Liberal Arts, Education, and the Sciences; Edgewood College \\ tma@edgewood.edu
}

Mark Vincent B. Yu

School of Education, University of California, Irvine

markv.yu@uci.edu

Stephanie Soto-Lara

School of Education, University of California, Irvine

ssotolar@uci.edu

\section{Sandra D. Simpkins}

School of Education, University of California, Irvine

simpkins@uci.edu

\begin{abstract}
Grounded in ecological frameworks, this study examines (a) the extent to which Latinx adolescents' perceptions of peer ethnic discrimination were associated with their participation in organized after-school activities, activity type, and ethnic composition; (b) different patterns of perceived peer ethnic discrimination; and (c) associations between discrimination patterns with key activity experiences including psychological engagement, perceived peer support, perceived leader support, and positive feelings in the activities. Using a pattern-centered approach, we applied latent profile analysis to analyze the data from 204 Latinx adolescents (53\% female, M age $=12.40)$ in Southwest United States. Latinx adolescents who did not participate in organized after-school activities perceived higher peer ethnic discrimination than Latinx adolescents who participated. Latinx adolescents who were the numerical ethnic majority in activities reported lower discrimination than those who were the numerical minority. Among those who participated, 4 patterns of peer ethnic discrimination Latinx adolescents experienced in activities were identified. These profiles included moderate discrimination (4\%), minimal discrimination (21\%), no discrimination (64\%), and somewhat negative beliefs (11\%), which were differentially related to adolescents' activity outcomes. Adolescents in the no discrimination group reported the most positive activity outcomes and those in the moderate discrimination group reported the most negative activity experiences. Adolescents who experienced little discrimination but felt other peers held negative beliefs
\end{abstract}

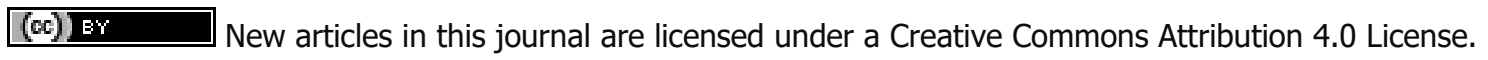
This journal is published by the University Library System, University of Pittsburgh and is cosponsored by the University of Pittsburgh Press. The Journal of Youth Development is the official peer-reviewed publication of the National Association of Extension 4-H Youth Development Professionals and the National AfterSchool Association. 


\section{Peer Ethnic Discrimination in Organized Activities}

about their ethnicity reported significantly lower psychological engagement and peer support than the no discrimination group. These findings highlight the importance of examining adolescents'varying patterns of perceived ethnic discrimination in activities and provides ways that activity practitioners can optimize organized activity settings for Latinx adolescents.

Key words: discrimination, Latinx youth, organized activities, after-school activities, extracurricular

\section{Introduction}

Participation in organized after-school activities has steadily increased over the years (Afterschool Alliance, 2014) and is linked to key indicators of positive youth development (e.g., social-emotional learning skills; Hurd \& Deutsch, 2017). High-quality organized after-school activities can promote positive youth development. However, recent qualitative research suggests that some adolescents from racial/ethnic minority groups experience discrimination, exclusion, microaggressions, and a lack of support in these settings (Gast et al., 2017; Lin et al., 2016; Ngo, 2017).

Although scholars have argued the need to examine the role of race, ethnicity, and culture in these settings, very few studies address these issues and how it may influence adolescents' experiences (Simpkins et al., 2017; Williams \& Deutsch, 2016). Preliminary research suggests that social-cultural factors related to race and ethnicity, such as peer ethnic discrimination, can influence adolescents' experiences in these settings (Echols \& Graham, 2018; Fredricks \& Simpkins, 2014). It is projected that by 2050, over half of all children under age 18 in the United States will be people of color (Federal Interagency Forum on Child and Family Statistics, 2016). Thus, understanding how to optimize organized activity settings for an increasingly diverse adolescent population is imperative.

Latinx adolescents are the fastest growing population in the U.S. school system (National Center for Education Statistics, 2015). Although not all Latinx adolescents experienced peer ethnic discrimination in activities, some have experienced micro-aggressions in the form of ethnic teasing and implicit stereotyping from peers and activity leaders (Lin et al., 2016). Given a lack of quantitative studies examining this topic, the aim of this study is to investigate the different patterns of peer ethnic discrimination profiles Latinx adolescents experienced in activities, and the links of these ethnic discrimination patterns to adolescents' engagement and experiences. 


\section{Peer Ethnic Discrimination in Organized Activities}

\section{Ecological Frameworks}

To effectively examine the processes underlying the effects of peer ethnic discrimination, both individual and contextual factors should be considered (Benner \& Graham, 2011). According to Bronfenbrenner's ecological theory, the reciprocal interactions between individuals and the people in individuals' immediate contexts, also known as proximal processes, are the space in which development transpires (Bronfenbrenner \& Morris, 1998). These proximal processes are situated within a series of nested contexts, ranging from immediate microsystems to more distal contexts. In this study, peer groups are viewed as proximal settings that are situated within the larger activity context. According to positive youth development perspectives (Lerner et al., 2017), positive developmental processes will emerge when the interplay between individuals and their immediate contexts or microsystems are positive and build on individuals' strengths. In contrast, contexts that do not build on individuals' strengths can give rise to negative developmental processes. Though organized after-school activities have been touted by scholars as a context for positive youth development, it is important to also understand when such contexts may, in fact, be detrimental because the context does not build on individuals' strengths.

A complementary ecological framework, the integrative model of minority youth development (García Coll et al., 1996), underscores critical aspects of the environment that may have profound influences in the developmental experiences of minoritized adolescents. This process is iterative and operates at multiple levels but is most represented in reciprocal influences between individuals' social position factors (e.g., race, ethnicity, social class) and their promoting/inhibiting contexts (García Coll et al., 1996). According to this framework, Latinx adolescents who perceive and/or experience ethnic discrimination may recognize their proximal contexts as unwelcoming. As a result, Latinx adolescents may be less likely to engage with and benefit from the experiences related to that context. Specific to this study, this framework helps to identify the potential mechanisms by which peer ethnic discrimination might affect Latinx adolescents' well-being. These discriminatory experiences can vary and create inhibiting organized activity contexts, which can lead to negative developmental outcomes for Latinx adolescents.

Recently, scholars have pulled together ecological theory, positive youth development, and theories like the integrative model of minority youth development to describe how race and ethnicity might shape the proximal processes within organized after-school activities (Simpkins et al., 2017; Williams \& Deutsch, 2016). This work underscores that the field needs to understand the specific ways organized activities not only support positive youth development 


\section{Peer Ethnic Discrimination in Organized Activities}

for all, but also provide culturally responsive practices to target issues related to peer ethnic discrimination (Simpkins et al., 2017). Doing so can create more inclusive activities that are both effective and responsive to all adolescents.

\section{Peer Ethnic Discrimination During Adolescence}

Peer ethnic discrimination involves differential behavioral acts including non-verbal (e.g., negative beliefs), verbal (e.g., teasing), and physical harassment from peers because of one's ethnic group membership (Brown \& Bigler 2005; Johnston \& Delgado, 2004). This type of discrimination taps into feeling disrespected, excluded, and insulted by peers (Johnston \& Delgado, 2004). The prevalence of racial/ethnic discrimination in the United States has been on the rise (Lee et al., 2019) and is a pervasive stressor in the daily lives of many racial/ethnic minority youth (Fisher et al., 2000). A recent meta-analysis of over 200 studies found that greater perceptions of ethnic discrimination were consistently linked to poorer adjustment across academic (e.g., achievement), social-emotional (e.g., self-esteem), and behavioral domains (e.g., risky behaviors) during adolescence (Benner et al., 2018). Evidence suggests that the rate at which adolescents report experiencing peer ethnic discrimination and the repercussions varies by their racial/ethnic background. Greene et al. (2006) found that Latinx (with the exception of Puerto Ricans) and Asian American adolescents tended to report higher levels of discrimination compared to other ethnic groups during the beginning of high school. In addition, Latinx adolescents tended to exhibit higher levels of mental health issues than their White and African American peers in response to discrimination (Benner et al., 2018). The aforementioned findings from various studies suggest the importance of examining discrimination experiences of Latinx adolescents.

Adolescents' experiences with discrimination are quite varied and subgroups are found in these experiences. Moreover, the various patterns (i.e., subgroups) in adolescents' perceived discrimination are found to be associated with different adolescent adjustment outcomes (Byrd \& Andrews, 2016; Garnett et al., 2014; Lorenzo-Blanco et al., 2016; Niwa et al., 2014; Nylund, Bellmore, et al., 2007). For example, Byrd and Andrews (2016), identified four unique patterns of perceived discrimination: highly discriminated group across multiple school personnel (14\%), highly discriminated group except by teachers $(8 \%)$, primarily discriminated by peers $(18 \%)$, and low discrimination group (59\%). Among these groups, the low discrimination group perceived the most positive school climate, teacher relationship, and engagement. In addition, different negative outcomes were observed between those who reported some and high discrimination. Despite research efforts to describe various discrimination profiles adolescents 


\section{Peer Ethnic Discrimination in Organized Activities}

may experience, such an examination focusing on Latinx adolescents remains absent. Exploring the heterogeneity within the construct of peer ethnic discrimination is an important step to uncovering meaningful profiles among Latinx adolescents who experience different forms of discrimination (e.g., negative beliefs, exclusion).

\section{Latinx Adolescents' Peer Relational Experiences in Organized Activities}

Positive peer relationships between adolescents in organized activities may be highly influential in the positive development of adolescents (Fredricks \& Simpkins, 2014). Adolescents who participate in high-quality organized activities and have a sense of connectedness with their peers report higher academic engagement and achievement and fewer social-emotional problems than their peers (Knifsend et al., 2018; Smith et al., 2013). Moreover, participating in activities and having supportive peer relationships in these settings can promote adolescents' prosocial behaviors and the formation of new friendships (Schaefer et al., 2011).

Although adolescents typically report positive experiences in organized activities, some adolescents report negative experiences based on their racial/ethnic group membership. For example, Lin and colleagues (2016) found that Latinx adolescents experienced ethnic discrimination in activities including overt (e.g., ethnic teasing) and covert forms of discriminatory behavior (e.g., implicit ethnic stereotypes). In another study, ethnographers found that recent Mexican immigrant adolescents believed they were being excluded from activities by their U.S.-born Mexican-descent peers because they did not speak English well and were perceived as being too "Mexican" (Bejarano, 2005). These peer-related incidents can lead to feelings of disrespect (Ettekal et al., 2015) and be among the reasons why Latinx adolescents leave or do not join activities (Simpkins et al., 2013). Although some evidence suggests that experiences of peer ethnic discrimination decline as adolescents age (Bellmore et al., 2012; Nishina \& Bellmore, 2010), no research to our knowledge has quantitatively examined adolescents' experiences concerning peer ethnic discrimination in organized activities.

Such a lack of attention has made it difficult for programs and activity leaders to understand the prevalence, as well as to conceptualize the ramifications and negative consequences of ethnic discrimination. Experiences of racial/ethnic discrimination is detrimental to Latinx adolescents' positive development, particularly when others within the environment, including adults, are not responsive to adolescents' discriminatory treatment from peers (García Coll et al., 1996). Similar to the school context, negative peer group dynamics, including peer ethnic discrimination, contribute to adolescent outcomes in organized activities. These negative experiences can affect 


\section{Peer Ethnic Discrimination in Organized Activities}

adolescents' psychological engagement (Dawes \& Larson, 2011), relations and interactions with adults (Gutiérrez et al., 2017), sense of belonging (Davalos et al., 1999), and the extent to which they perceive activities as emotionally supportive and safe (Dworkin \& Larson, 2007).

Given that ethnic discrimination can be both explicit and implicit (Lin et al., 2016), adolescents may not experience the same pattern or type of ethnic discrimination. For example, an adolescent who experiences negative stereotypes about his/her ethnicity may not necessarily get excluded by peers in activities. It is even possible that adolescents' activity engagement and experiences depend on their pattern of ethnic discrimination. For example, adolescents who experience only one form of ethnic discrimination may have different activity outcomes from those who experience multiple forms of discrimination. This study employs both variable- and pattern-centered approaches to provide a comprehensive understanding of Latinx adolescents' peer ethnic discrimination.

\section{Current Study}

Overall, the documented deleterious effects of peer ethnic discrimination suggest the need for close attention to peer dynamics that reflect Latinx adolescents' participation and varied experiences in activities. To this end, the present study examines three research questions: First: Is Latinx adolescents' overall perceived level of peer ethnic discrimination associated with activity indicators, including participation status, activity types, and ethnic compositions in the school and in the activity (i.e., perceived numerical ethnic minority status), and with participant demographics, including gender and nativity status? Second: What are different patterns of Latinx adolescents' self-reported peer ethnic discrimination in organized after-school activities? Third: To what extent do peer ethnic discrimination patterns predict key activity outcomes including psychological engagement, perceived peer support, perceived leader support, and positive feelings in the activity? We believed that, to this topic, both variable-centered and pattern-centered methods help to maximize the understanding of Latinx adolescents' experienced discrimination in the activity.

First, a variable-centered approach is used to investigate the first research question so we can easily compare the mean differences in discrimination across critical activity indicators (e.g., the numeric minority status, activity type, activity participation status). The integrative model of minority youth development (García Coll et al., 1996) and qualitative findings show that Latinx adolescents can experience discrimination in activities including microaggressions and offensive remarks from peers and adult staff based on their ethnic culture (e.g., Gutiérrez et al., 2017; 


\section{Peer Ethnic Discrimination in Organized Activities}

Lin et al., 2016). These negative culture-related experiences can undermine Latinx participation in activities (see Erbstein \& Fabionar, 2019 for a review). Thus, we expect that Latinx adolescents who did not participate in activities report higher perceived discrimination than adolescents who participated. We also expect Latinx adolescents who are the numerical ethnic majority (i.e., perceived more proportion of Latinx adolescents in the activity) will perceive less discrimination than those who are the numerical minority in the activity (Bellmore et al., 2012; Borden et al., 2005).

Then, our second and third research questions are an extension of previous studies that have conceptualized peer ethnic discrimination as a single construct (e.g., Benner \& Graham, 2011). Pattern-centered approaches (e.g., latent profile analysis) are suitable for our aim to identify the different patterns of peer ethnic discrimination reported by Latinx adolescents (Nylund, Asparouhov, \& Muthén, 2007; Nylund, Bellmore, et al., 2007). A pattern-centered approach is useful because it does not require specification of a priori groups (Nylund, Asparouhov, \& Muthén, 2007). Yet, aligned with prior research on ethnic discrimination outside the setting of organized activities (Byrd \& Andrews, 2016; Garnett et al., 2014; Lorenzo-Blanco et al., 2016), we expect to identify at least three profiles: low discrimination, high discrimination, and a moderate group who experienced a mix of high and low scores across several discrimination indicators. In addition, based on prior research suggesting significant adolescent outcome differences experienced by discrimination subgroups (e.g., Lorenzo-Blanco et al., 2016), we expect that adolescents who have low patterns of discrimination will report the highest activity engagement and positive experiences. We also expect adolescents who have moderate or high discrimination will report low activity engagement and negative experiences.

\section{Method}

\section{Participants}

The participants were 204 Latinx adolescents ( $53 \%$ female, Mage $=12.40$ years, $S D=.57$ ) in seventh grade from four middle schools in the Southwest. The four selected schools varied in ethnic composition ( $16 \%$ to $91 \%$ Latinx) and family socioeconomic status ( $21 \%$ to $93 \%$ students with free or reduced-price lunch). Across the four schools, adolescents were selected based on their participation in organized after-school activities comprised of three groups: (a) students who participated in a community-based activity $(11 \%)$, (b) students who participated in a school-based activity (69\%), and (c) students who did not participate in any organized activity at recruitment (20\%). Adolescents who participated in an organized after-school activity, community or school-based, participated in one of four main types of activities offered 


\section{Peer Ethnic Discrimination in Organized Activities}

at the four schools: sports (29\%, $n=45$; e.g., soccer, cross-country, basketball); art (38\%, $n=$ 59; e.g., band, chorus, orchestra); leadership/academic activities (14\%, $n=22$; student council, book club, newspaper); and community-based activities (18\%, $n=28$; e.g., youth group, Boy Scouts, young woman religious). Table 1 shows the distribution of the four types of activities reported by the study participants across the four schools. The independent chi-square test showed activity types reported did not significantly vary by school: chi-square $(12)=$ $14.40, p=.276$.

Table 1. School by Activity Type Crosstabulation

\begin{tabular}{|l|c|c|c|c|c|}
\hline \multicolumn{2}{|c|}{ Activity types } \\
\hline Schools & $\begin{array}{c}|c| \\
\text { academic }\end{array}$ & Art & Sport & Community & No activity \\
\hline School 1 & $4(14 \%)$ & $8(27 \%)$ & $9(31 \%)$ & $4(14 \%)$ & $4(14 \%)$ \\
\hline School 2 & $9(13 \%)$ & $14(20 \%)$ & $17(25 \%)$ & $8(12 \%)$ & $21(30 \%)$ \\
\hline School 3 & $3(6 \%)$ & $18(39 \%)$ & $5(11 \%)$ & $10(22 \%)$ & $10(22 \%)$ \\
\hline School 4 & $6(10 \%)$ & $19(32 \%)$ & $14(23 \%)$ & $6(10 \%)$ & $15(25 \%)$ \\
\hline
\end{tabular}

From the overall study sample, 75\% ( $n=154)$ of adolescents participated in at least one activity whereas $25 \%$ ( $n=50$ ) of adolescents did not participate in an organized activity. Active and non-active Latinx adolescents within each school were matched on characteristics that predicted participation in organized activities, such as grade point average, language preference, and nativity (Mahoney et al., 2009). Data from 204 Latinx adolescents were used to examine Research Question 1, and data from 154 Latinx adolescents who participated in an activity were used in our second research question.

\section{Procedures}

The research team sent letters home with the adolescents describing the study. Families who returned the letters noting their interest in the study were contacted. Of the families who returned interest forms ( $n=827), 81 \%(n=667)$ agreed to participate in the study. The 667 participants were stratified by school, specific activity, gender, race, and ethnicity. For this study, the 204 Latinx adolescents were purposely selected from the various strata to balance gender, to ensure participation in each type of activity, and to ensure that each specific activity was offered in at least two of the four schools. 


\section{Peer Ethnic Discrimination in Organized Activities}

Parental consent and adolescent assent forms were obtained. Quantitative survey data was collected through phone interviews. Phone interviews lasted approximately 60 to 90 minutes and assessed their perceived peer ethnic discrimination at the activity (and projected discrimination for adolescents of non-active status), psychological engagement, perceived activity experiences, in the activity, and demographic information. Bilingual interviewers conducted interviews in participants' preferred language. Adolescent interviews were conducted in English except for one in Spanish. Surveys were translated by Spanish-fluent research assistants and checked for accuracy using a forward-translation and panel/group method approach (Knight et al., 2009).

\section{Measures}

Participants were instructed to report their perceived peer ethnic discrimination, activity experiences, and engagement about one single activity in order to reduce participant burden.

\section{Perceived Peer Ethnic Discrimination}

Latinx adolescents' perceived discrimination from peers was assessed with the Perceptions of Activity Discrimination scale, which was adapted from Johnston and Delgado's (2004) Perceptions of School Discrimination Scale. This scale includes six items for peer ethnic discrimination both for those who participated and who did not participate. Adolescents who attended an activity were asked to describe their perceived discrimination whereas adolescents who did not attend the activity were asked to think about how they thought Latinx adolescents are treated in organized activities. The differences in wordings were noted in the parentheses. For examples, "The kids at the organized activity have negative beliefs about teen's ethnicity that affect the way they treat (you/teens); the kids at the organized activity would exclude (you/teens) from things they do outside the activity because of (your/teens') ethnicity." The full item description can be found in Table 2. The scale was assessed on a 5-point Likert scale (i.e., $0=$ strongly disagree, to $2=$ in the middle, to $4=$ strongly agree) and has the alpha $=.90$.

\section{Activity Engagement}

Adolescents' engagement at organized activities was measured by the Psychological Engagement Scale from Moore and Hansen (2012) that consisted of six items. These items assessed how engaged the teenager felt when they were at their activity such as if they felt like they were working towards an important goal, were not bored, and feeling challenged (e.g., "There are always things I'm trying to work on and achieve in this activity; " 0 =strongly disagree to, 2 = in the middle, to 4 = strongly agree). The reliability across the 6 items is .68. 


\section{Peer Ethnic Discrimination in Organized Activities}

\section{Experiences at Activities}

Adolescents' experiences at activities were assessed by a measure from Rosenthal and Vandell (1996) to capture adolescents' experiences with peers and staff at their activity. The measure includes 11 items that measured perceived peer support and leader support. The perceived peer support assessed the extent adolescents positively affiliate with other adolescents in the activities (5 items, e.g., "I have a lot of friends there; I have a good time playing with other kids there"; 0 = never, 2 = sometimes, 4 = always; alpha=.75). The perceived leader support assessed the extent adolescents positively interact with the activity leaders ( 6 items, e.g., "I can tell the leaders there about my problems if I need to; the leader there cares about me"; alpha=.70). Latinx adolescents also reported their positive emotional experiences in their activity (three items, e.g., Tell me how often "you feel happy when you are at the activity", $0=$ never, 4 = always; Shernoff \& Vandell, 2007). Items were averaged to create an indicator of Latinx adolescents' positive feelings about the activity (alpha $=.75$ ).

\section{Adolescents' Demographics}

Nativity status ( 1 = born outside the U.S., 0 = born in the U.S.) was reported by adolescents where $84 \%$ ( $n=129)$ reported being born in the U.S. and $16 \%(n=25)$ reported being born outside the U.S. In addition, gender ( $1=$ female, $0=$ male) was reported by adolescents, where $59 \%(n=90)$ reported being female and $41 \%(n=63)$ reported being male. One adolescent preferred not to answer. The ethnic-composition of the school was computed based on Simpson's diversity index and we assigned the status based on numeric ethnic status within schools. The numerical majority was assigned if a school consists of more than $50 \%$ Latinx adolescents ( 1 = Latinx numeric-majority; 0 = Latinx numeric-minority). The ethnic-composition of the activity was assessed by a single item asking adolescents' perception of the proportion of Latinx (because our large sample contains either White or Latinx) adolescents in activity (i.e., "How many of the teenagers at the activity are White?"; $0=$ none, $1=$ less than half, $2=$ about half, $3=$ more than half, $4=$ all of them). A higher score indicates that the activity had fewer Latinx adolescents (i.e., perceived numerical ethnic minority).

\section{Data Analysis}

Models were estimated in both SPSS Version 24 and in Mplus Version 8 using full information maximum likelihood estimation with robust SEs (MLR; Muthén \& Muthén, 1998-2015). To examine the first research question, we used a series of $t$-tests and ANOVA to compare the 


\section{Peer Ethnic Discrimination in Organized Activities}

mean scores of peer ethnic discrimination across participation status (yes or no), ethnic composition in the school and in the activity, gender, and nativity status.

To examine our second research question, we explored Latinx adolescents' experiences with peer ethnic discrimination for those who participated in the activity with latent profile analysis (LPA). We first specified models of varying number of classes. To determine the optimal solution, we examined multiple fit indices including the Bayesian Information Criterion (BIC), Akaike's Information Criterion (AIC), Consistent Akaike's Information Criterion (CAIC), samplesize adjusted BIC (saBIC), Lo-Mendell-Rubin adjusted Likelihood Ratio Test (LMR-aLRT), Approximate Weight of Evidence Criterion (AWE), and the approximate correct model probability (cmP).

Smaller AIC, BIC, ACAIC, and saBIC values indicate better fitting solutions (Collins \& Lanza, 2010). Significant $p$ values of LMR-aLRT indicate that the model with $k$ classes has better fit to the data than a model with $k-1$ classes. A cmP value closer to 1 indicates that actual probability of Model $A$ being the correct model relative to a set of J models under consideration (Masyn, 2017).

Our third research question compared means of activity outcomes (e.g., psychological engagement) for statistically significant differences. The Wald chi-square test was used to assess differences in distal means between pairs of profile membership.

\section{Results}

Preliminary analyses were performed that included descriptive statistics and bivariate correlations (see Table 2). The bivariate correlations indicated that all peer ethnic discrimination items were positively correlated with each other, and they were negatively correlated with adolescents' activity engagement and positive experiences. There were no missing data on any of the outcome variables and only $1.5 \%(n=3)$ missing on all peer ethnic discrimination items. The three participants who did not fill the discrimination items did not participate in an organized activity, so their data was not used in the latent profile analysis. All items have kurtosis and skewness below 2.0 indicating that the data were normally distributed. 
Table 2. Correlations and Descriptive Statistics of Key Variables

\begin{tabular}{|c|c|c|c|c|c|c|c|c|c|c|}
\hline Variables & 1. & 2. & 3. & 4. & 5. & 6. & 7. & 8. & 9. & 10. \\
\hline \multicolumn{11}{|l|}{ Peer ethnic discrimination items } \\
\hline $\begin{array}{l}\text { 1. Peer held negative belief } \\
\text { toward you bc of ethnicity. }\end{array}$ & - & $.62 * *$ & $.44 * *$ & $.53^{* *}$ & $.43 * *$ & $.53 * *$ & $-.18^{*}$ & $-.30 * *$ & $-.28 * *$ & -.14 \\
\hline $\begin{array}{l}\text { 2. Being excluded bc of } \\
\text { ethnicity. }\end{array}$ & & - & $.65 * *$ & $.69 * *$ & $.68^{* *}$ & $.65^{* *}$ & $-.27 * *$ & $-.27 * *$ & $-.28 * *$ & $-.25^{* *}$ \\
\hline $\begin{array}{l}\text { 3. Being called name bc of } \\
\text { ethnicity. }\end{array}$ & & & - & $.54 * *$ & $.66^{* *}$ & $.58 * *$ & $-.29 * *$ & $-.17^{*}$ & $-.27 * *$ & $-.30 * *$ \\
\hline $\begin{array}{l}\text { 4. Peer assumed you not as } \\
\text { smart bc of ethnicity. }\end{array}$ & & & & - & $.68^{* *}$ & $.69 * *$ & $-.20 *$ & $-.21^{*}$ & $-.21 *$ & $-.18^{*}$ \\
\hline $\begin{array}{l}\text { 5. Peer did not hang out with } \\
\text { you bc of ethnicity. }\end{array}$ & & & & & - & $.77 * *$ & -.14 & -.14 & $-.18^{*}$ & $-.24 * *$ \\
\hline $\begin{array}{l}\text { 6. Was treated badly bc of } \\
\text { ethnicity. }\end{array}$ & & & & & & - & $-.16^{*}$ & $-.16^{*}$ & $-.25 * *$ & $-.20 *$ \\
\hline 7. Psychological engagement & & & & & & & - & $.45^{* *}$ & $.48^{* *}$ & $.34 * *$ \\
\hline 8. Perceived peer support & & & & & & & & - & $.50 * *$ & $.39 * *$ \\
\hline 9. Perceived leader support & & & & & & & & & - & $.39 * *$ \\
\hline 10. Positive feelings & & & & & & & & & & - \\
\hline Mean & .59 & .38 & .38 & .42 & .29 & .31 & 3.08 & 3.29 & 3.19 & 3.10 \\
\hline$S D$ & .70 & .57 & .55 & .65 & .54 & .53 & .48 & .54 & .55 & .66 \\
\hline
\end{tabular}

Note. Variables 1 to 6 are the perceived peer ethnic discrimination items in the organized activities. Variables 7 to 10 are the activity outcomes and experiences reported by Latinx adolescents. bc=because. 


\section{Peer Ethnic Discrimination and Activity Participation}

First, the extent to which active and non-active Latinx adolescents differed in their perceived peer ethnic discrimination was examined. Latinx adolescents who did not participate in organized activities reported higher levels of perceived peer ethnic discrimination, $n=47, M=$ $0.65, S D=0.53$, than those who participated in organized activities, $n=154, M=0.40$, $S D=0.48, t(199)=3.03, p=.003, d=.49$. Moreover, the extent to which activity type was statistically related to perceived peer ethnic discrimination for those who actively engaged in organized activities was examined. One-way ANOVA revealed that there were no significant differences among the four activity types (i.e., club, art, sport, community) in the mean differences of peer ethnic discrimination, $f(3,150)=1.058, p=.37, \eta^{2}=.02$. In addition, Latinx adolescents' peer ethnic discrimination was associated with having fewer Latinx adolescents in the activity $(r=.19, p<.001)$. The peer ethnic discrimination did not differ by ethnic composition of the school, $t(199)=-1.14, p=.26, d=.03$, gender, $t(198)=-.11, p=$ $.91, d=.02$, nor by nativity status, $t(197)=.33, p=.74, d=.06$.

\section{Identifying the Patterns of Adolescents' Perceived Peer Ethnic Discrimination}

To identify underlying patterns of peer ethnic discrimination experienced by Latinx adolescents, LPA was performed. Table 3 shows all model fit indices for the models with one to five classes. There is a relative smaller decrease of AIC, CAIC, BIC, saBIC, and AWE for models with four or more classes, and the results of $\mathrm{cmP}$ also indicated the four-class solution appeared to be the best fitting models among one to five classes. When we compared five-class with the four-class model, the additional class identified in the five-class model was similar to other classes and not statistically distinct (e.g., two classes in this case represents high discrimination group, in which one class contained only one participant).

Table 3. Model Fit Indices for Latent Profile Analyses

\begin{tabular}{|l|c|c|c|c|c|c|c|c|}
\hline Model & LogL & AIC & CAIC & BIC & saBIC & AWE & $\begin{array}{c}\text { LMR- } \\
\text { aLRT }\end{array}$ & cmP(k) \\
\hline 1-class & -813.83 & 1651.66 & 1700.10 & 1688.10 & 1650.12 & 1784.54 & -- & 0.00 \\
\hline 2-class & -813.83 & 1665.66 & 1742.36 & 1723.36 & 1580.44 & 1876.06 & 595.49 & 0.00 \\
\hline 3-class & -507.64 & 1067.28 & 1172.24 & 1146.24 & 950.68 & 1355.21 & 137.00 & 0.00 \\
\hline 4-class & -338.69 & 743.37 & 876.59 & 843.59 & 595.37 & 1108.81 & 191.75 & 1.00 \\
\hline 5-class & -284.40 & 648.80 & 810.28 & 770.28 & 469.40 & 1091.76 & 105.42 & 0.00 \\
\hline
\end{tabular}


Thus, the four-class model was selected as the most parsimonious solution and consisted of $4 \%$ of adolescents who showed a somewhat middle score for discrimination in the activities (meaning they did not disagree on being discriminated), especially on items of judged not as smart or being excluded based on their ethnicity (labeled moderate discrimination), 21\% of those who somewhat disagree on all discrimination items (labeled minimal discrimination), 64\% those who strongly disagreed on all discrimination items (labeled no discrimination), and $11 \%$ of those who strongly disagree on most of the discrimination items but showed somewhat middle scores on the item of perceived negative beliefs (related to ethnicity) held by the peers in particular (labeled possible somewhat negative beliefs). See Figure 1 for the four latent profiles.

Figure 1. Latent Profile Analysis Solutions for Perceived Peer Ethnic Discrimination of Latinx Adolescents Who Participated in the Activities

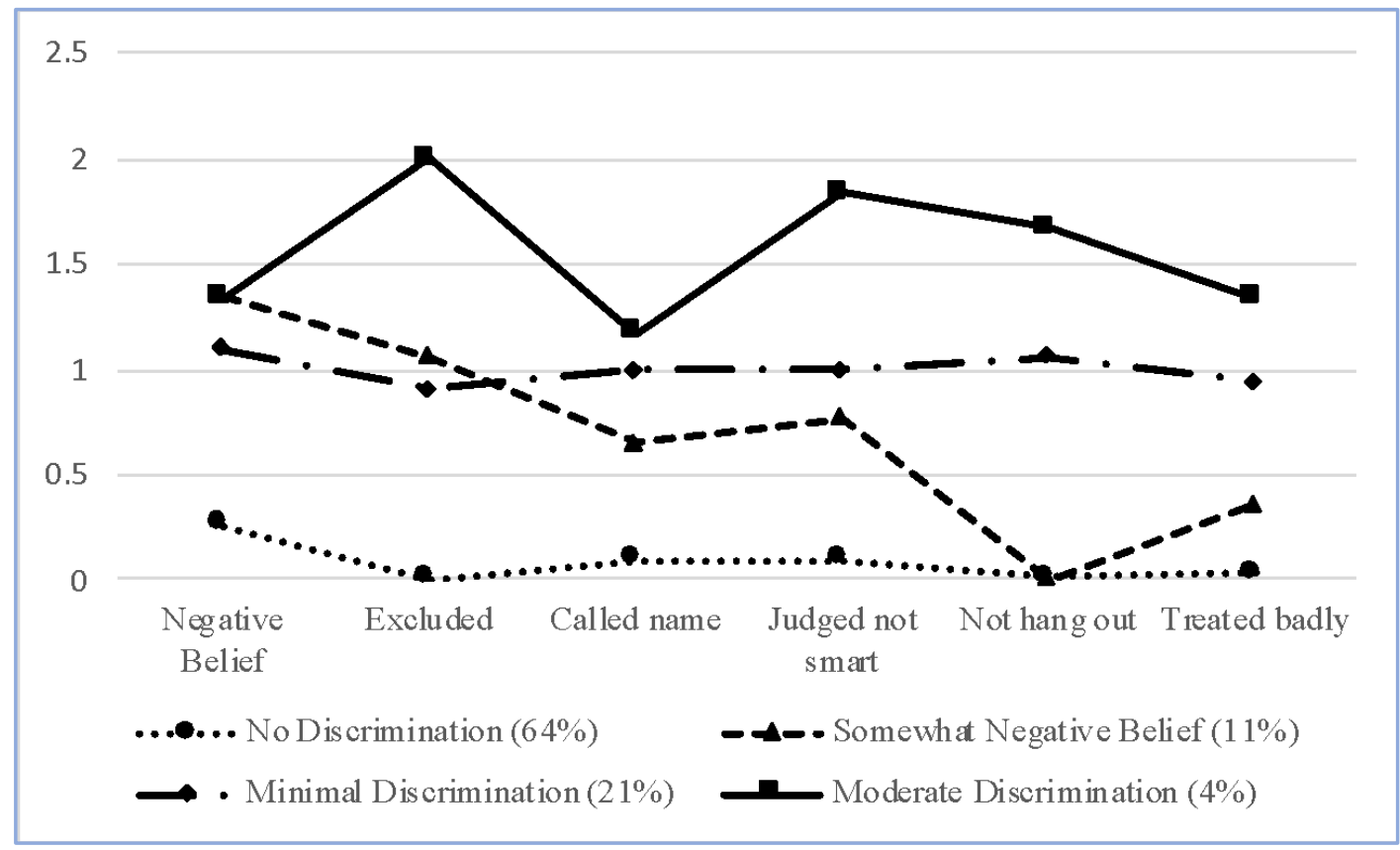

The $y$-axis represents the scale scores. We also examined if Latinx adolescents who were grouped into the profiles were different in terms of demographic variables that included gender $\left(\chi^{2}(3)=1.6, p=.66\right.$, phi $=.10)$, nativity status $\left(x^{2}(3)=3.5, p=.32\right.$, phi $\left.=.15\right)$, activity types $\left(x^{2}(9)=9.2, p=.42\right.$, phi $\left.=.24\right)$, ethnic composition of the school $\left(\chi^{2}(3)=1.28, p=.73\right.$, phi=.09), and ethnic composition in the activity, $\left(F(3,150)=.47, p=.66, \eta^{2}=.02\right)$ and found no significant differences. 


\section{Peer Ethnic Discrimination in Organized Activities}

As a validity check, we examined whether the four profiles were statistically distinguishable on the ethnic discrimination items. Table 4 shows the descriptive differences in the six peer ethnic discrimination indicators among the four discrimination profiles. The results showed our profiles were distinguishable among most of the peer ethnic discrimination items, except for the item about negative belief where only three (out of six total) pairs of statistical differences were identified.

Table 4. Descriptive Differences in Perceived Ethnic Discrimination Items by Profile Membership

\begin{tabular}{|c|c|c|c|c|c|}
\hline \multirow[b]{2}{*}{ Peer ethnic discrimination } & \multicolumn{4}{|c|}{ Perceived ethnic discrimination profiles } & \multirow[b]{2}{*}{$\begin{array}{l}\text { Statistical } \\
\text { significance }\end{array}$} \\
\hline & $\begin{array}{c}\text { No } \\
\text { discrimination }\end{array}$ & $\begin{array}{l}\text { Somewhat } \\
\text { negative }\end{array}$ & $\begin{array}{c}\text { Minimal } \\
\text { discrimination }\end{array}$ & $\begin{array}{c}\text { Moderate } \\
\text { discrimination }\end{array}$ & \\
\hline & $M(S D)$ & $M(S D)$ & $M(S D)$ & $M(S D)$ & $F_{(3,150)}$ \\
\hline $\begin{array}{l}\text { Peer held negative belief } \\
\text { because of ethnicity }\end{array}$ & $0.25(.50)^{\mathrm{a}}$ & $1.35(.78)^{\mathrm{b}}$ & $1.09(.46)^{b}$ & $1.33(.51)^{b}$ & $38.25^{* * *}$ \\
\hline Excluded because of ethnicity & $0(.00)^{\mathrm{a}}$ & $1.06(.24)^{b}$ & $0.91(.29)^{c}$ & $2.00(.00)^{d}$ & $638.48^{* * *}$ \\
\hline $\begin{array}{l}\text { Called names because of } \\
\text { ethnicity }\end{array}$ & $0.09(.28)^{\mathrm{a}}$ & $0.65(.49)^{b}$ & $1.00(.44)^{c}$ & $1.17(.75)^{\mathrm{c}}$ & $61.01^{* * *}$ \\
\hline $\begin{array}{l}\text { Assumed not smart because of } \\
\text { ethnicity }\end{array}$ & $0.09(.32)^{a}$ & $0.76(.75)^{\mathrm{b}}$ & $1.00(.50)^{b}$ & $1.83(.40)^{c}$ & $63.21^{* * *}$ \\
\hline $\begin{array}{l}\text { Don't hang out with because of } \\
\text { ethnicity }\end{array}$ & $0.01(.10)^{\mathrm{a}}$ & $0(.00)^{\mathrm{a}}$ & $1.06(.24)^{b}$ & $1.67(.51)^{c}$ & $427.251^{* * *}$ \\
\hline $\begin{array}{l}\text { Treated you badly because of } \\
\text { ethnicity }\end{array}$ & $0.03(.53)^{\mathrm{a}}$ & $0.35(.17)^{\mathrm{b}}$ & $0.94(.60)^{c}$ & $1.33(.43)^{d}$ & $83.301^{* * *}$ \\
\hline
\end{tabular}

Note. The means in each row that share the same superscripts are not significantly different from each other. The means that have different superscripts in the same row are significantly different at $p<.001$. ${ }^{*} p<.05 . * * p<.01 . * * * p<.001$.

\section{The Peer Ethnic Discrimination Profiles Predicting Activity Outcomes}

The third research question was to test the extent to which the patterns of Latinx adolescents' perceived peer ethnic discrimination were associated with their activity engagement and experiences. The three-step method was used to test differences across the different groups. Table 5 includes the estimates along with the Wald chi-square test results. In this analysis, the no ethnic discrimination group was used as the reference class as it consists of the most Latinx 


\section{Peer Ethnic Discrimination in Organized Activities}

adolescent sample in our study and therefore may represent a normative class. Means for the activity outcomes were estimated for each class and compared for statistically significant differences. Gender was originally controlled in the model; however, because of the nonsignificant associations between gender and any other variables in the model, for parsimonious purposes, we decided to remove gender from our final model reported here.

\section{Table 5. Estimates of Peer Ethnic Discrimination Patterns to Activity Outcomes}

\begin{tabular}{|l|c|c|c|c|c|}
\hline & $\begin{array}{c}\text { Moderate } \\
\text { discrimination }\end{array}$ & $\begin{array}{c}\text { Minimal } \\
\text { discrimination }\end{array}$ & $\begin{array}{c}\text { Somewhat } \\
\text { negative beliefs }\end{array}$ & $\begin{array}{c}\text { No } \\
\text { discrimination }\end{array}$ & $\begin{array}{c}\text { Wald } \\
\text { chi-square test }\end{array}$ \\
\hline $\begin{array}{l}\text { Activity } \\
\text { outcomes }\end{array}$ & Est $(S E)$ & Est $(S E)$ & Est $(S E)$ & Est $(S E)$ & Chi-square $(d f)$ \\
\hline $\begin{array}{l}\text { Psychological } \\
\text { engagement }\end{array}$ & $2.75(.18)^{\mathrm{a}}$ & $2.96(.07)^{\mathrm{a}}$ & $2.83(.11)^{\mathrm{a}}$ & $3.18(.05)^{\mathrm{b}}$ & $18.56(3)^{* * *}$ \\
\hline $\begin{array}{l}\text { Perceived peer } \\
\text { support }\end{array}$ & $2.91(.33)^{\mathrm{a}}$ & $3.22(.08)^{\mathrm{a}}$ & $2.99(.14)^{\mathrm{a}}$ & $3.39(.05)^{\mathrm{b}}$ & $14.79(3)^{* *}$ \\
\hline $\begin{array}{l}\text { Perceived } \\
\text { leader support }\end{array}$ & $2.67(.21)^{\mathrm{a}}$ & $3.03(.11)^{\mathrm{b}}$ & $3.02(.13)^{\mathrm{ab}}$ & $3.30(.05)^{\mathrm{c}}$ & $19.88(3)^{* * *}$ \\
\hline $\begin{array}{l}\text { Positive } \\
\text { feelings }\end{array}$ & $2.44(.20)^{\mathrm{a}}$ & $2.88(.11)^{\mathrm{b}}$ & $3.04(.12)^{\mathrm{bc}}$ & $3.23(.07)^{\mathrm{c}}$ & $20.89(3)^{* * *}$ \\
\hline
\end{tabular}

Note. Reference class is Low discrimination. Est = coefficient estimate. The coefficients within each row that have the same superscripts are not significantly different from each other but are significantly different from coefficients whose superscripts differ. Coefficients with two superscripts do not differ significantly from coefficients with either of those individual superscripts.

${ }^{*} p<.05$. ** $p<.01$. *** $p<.001$.

Latinx adolescents who were in the no discrimination group reported the highest scores on all of the outcomes, including psychological engagement, peer support, leader support, and positive feelings compared to the other three groups (i.e., minimal discrimination, somewhat negative belief, and moderate discrimination). In contrast, Latinx adolescents who were in the moderate discrimination group reported the worst scores on psychological engagement, perceived peer support, leader support, and positive feelings. Specifically, adolescents in the minimal discrimination group reported significantly less psychological engagement, peer support, leader support, and positive feelings compared to adolescents in the no discrimination group. This group also reported higher leader support and positive feelings than the moderate 


\section{Peer Ethnic Discrimination in Organized Activities}

discrimination group. Adolescents in the somewhat negative beliefs group reported less psychological engagement and perceived peer support compared to the no discrimination group, but they reported significantly higher positive feelings than the moderate discrimination group. They also did not differ from the minimal discrimination group in any of the activity outcomes. Adolescents in the no discrimination group reported significantly higher peer support than minimal discrimination group, but they did not differ significantly from somewhat negative belief group on leader support and positive feelings.

\section{Discussion}

During adolescence, Latinx adolescents begin to understand and consider the implications of how others view their racial/ethnic group (Umaña-Taylor et al., 2015). Thus, as they move through this developmental period, ethnic-based discrimination can represent a significant risk factor (García Coll et al., 1996). Further, given the importance placed on peer relationships during this period, ethnic discrimination by peers can be particularly detrimental (Greene et al., 2006). In line with this perspective, a plethora of studies have shown that peer ethnic discrimination in the school context has detrimental effects on Latinx adolescents' academic, social-emotional and behavioral development (e.g., Bellmore et al., 2012; Nishina \& Bellmore, 2010). Few, however, have examined these processes in organized after-school activities (e.g., Lin et al., 2016). Towards a better understanding of these processes, we examined Latinx adolescents' perceptions of peer ethnic discrimination and their participation and experiences in these settings.

\section{Peer Ethnic Discrimination and Activity Participation}

Our findings indicate that Latinx adolescents who participated in organized after-school activities perceived significantly less peer ethnic discrimination compared with those who did not participate in activities. Previous research on organized activities has suggested that adolescents may take into account the peer ethnic context when deciding to participate in activities (e.g., Simpkins et al., 2011; Borden et al., 2005). Further, if Latinx adolescents perceive these contexts as negative, hostile, or unwelcoming, they may be more likely to disengage (García Coll et al., 1996; Borden et al., 2005). In line with these studies, our findings suggest that peer group dynamics such as peer ethnic discrimination may be at play in adolescents' decision to participate or persist in activities. 


\section{Peer Ethnic Discrimination in Organized Activities}

However, it is essential to note that there are many reasons why adolescents do and do not participate in activities, which can vary based on their interests (Dawes \& Larson, 2011) or as a result of constraints (e.g., lack money/transportation, safety issues) (Borden et al., 2005). Further investigation is warranted to examine the factors that deter and prevent adolescents from participating in organized after-school activities. In addition, consistent with findings showing that ethnic-numeric status affected minority adolescents' experiences (Bellmore et al., 2012), we found that Latinx adolescents who were the numeric minority in activities perceived more peer ethnic discrimination compared to Latinx adolescents who were the numeric majority in activities. This result suggests the importance of ethnic cultural representation in activities in the context of a safe and inclusive program climate as a protective step to building a more welcoming setting for Latinx adolescents.

\section{Distinct Patterns of Peer Ethnic Discrimination}

Although Latinx adolescents who participated in organized after-school activities on average perceived low peer ethnic discrimination, the results revealed four distinct patterns: $64 \%$ of adolescents perceived no discrimination, $4 \%$ perceived moderate discrimination, $21 \%$ perceived minimal peer ethnic discrimination, and $11 \%$ perceived somewhat negative beliefs. Of these groups, the moderate discrimination group reported the least positive activity outcomes, which is consistent with literature that suggests peer ethnic discrimination is associated to adverse developmental outcomes (Benner et al., 2018; Garnett et al., 2014; Lorenzo-Blanco et al., 2016). Although the $21 \%$ of Latinx adolescents who perceived minimal discrimination were more likely to disagree on being discriminated across a number of indicators than the moderate discrimination group, they still experienced lower psychological engagement and low leader support and positive feelings in activities compared to the no discrimination group. These findings underscore the importance of attending to even a slight amount of peer ethnic discrimination within activities. That is, Latinx adolescents may be at risk of experiencing negative activity outcomes if they did not feel the activity atmosphere is completely free of discrimination.

Special attention should be given to adolescents in the subgroup labeled somewhat negative beliefs. These adolescents perceived significantly lower psychological engagement and perceived peer support compared to adolescents in the no discrimination group. In most cases, adolescents who fell in this subgroup may be overlooked and considered not to be affected by peer ethnic discrimination in activities because they socialize or "hang out" with their peers well and are not excluded by their peers in activities. Yet their lower psychological engagement in 


\section{Peer Ethnic Discrimination in Organized Activities}

the activity suggests that the negative consequences of peer ethnic discrimination may be possible even if the observable discriminatory behaviors are absent. Unfortunately, if researchers simply collapsed adolescents' experiences of ethnic discrimination components (from a scale) into a single variable, these adolescents who have had a low average score in discrimination composite scores may be easily overlooked.

The findings of this study suggest the importance of paying close attention to adolescents' varying experiences of peer ethnic discrimination in activities which include their perceptions of others' negative beliefs about their ethnic background. Indeed, previous research suggests that negative beliefs (e.g., stereotypes) about one's racial/ethnic group can have adverse effects on the psychological adjustment of ethnic minority adolescents (Brown \& Bigler, 2005; Fisher et al., 2000). Meanwhile, research also suggests that support from peers and leaders can buffer Latinx adolescents from the negative effects of discrimination (Degarmo \& Martinez, 2006). In line with a positive youth development perspective, findings of the present study suggest the importance of understanding the unique and varied cultural experiences and needs of Latinx adolescents in order to develop more effective and responsive practices that target the negative effects of ethnic discrimination in activities (Simpkins et al., 2017). Importantly, these efforts should go beyond identifying between-group racial/ethnic differences among adolescents towards a better understanding of within-group heterogeneity (e.g., gender, income, individual experiences) with respect to the promotion of positive youth development outcomes (Williams \& Deutsch, 2016).

\section{Study Limitations}

While the current study provides strong support for heterogeneity in adolescents' peer ethnic discrimination in activities, a number of limitations and future directions should be considered. First, the cross-sectional and correlational design of this study did not allow us to make causal inferences between peer ethnic discrimination and the activity experiences. Meanwhile, the study results cannot be generalized to adolescents who declined to participate in the study (19\%). Second, this research relies on self-report data from adolescents; thus, we did not have access to the fuller picture of the processes of adolescents' experiences of peer ethnic discrimination as triangulated by other perspectives including staff and more objective observers. Relatedly, we cannot completely exclude the possibility of adolescents underreporting their negative experiences in the activities. Previous research has found that adolescents are more likely to report salient experiences of discrimination (Huynh \& Fuligni, 


\section{Peer Ethnic Discrimination in Organized Activities}

2010). Moreover, this study did not measure gender and lived experience of activity leaders ${ }^{1}$, which may very likely impact adolescents' reported experiences in the activities. Lastly, we examined peer ethnic discrimination among Latinx adolescents, predominantly of Mexicandescent, and so these findings may not adequately describe the experience of other racial/ethnic groups.

\section{Areas for Future Research.}

Our findings align with prior research that have shown that people who tend to perceive more discrimination also express lower interest in activities (Seaton \& Yip, 2009). At the same time, it is possible that additional factors exist that influence both Latinx adolescents' perceptions of discrimination and negative experiences in activities. For example, there may be other factors (e.g., perceived staff ethnic discrimination) in combination with peer ethnic discrimination that influence adolescents' negative experiences in the activities. Additionally, adolescents' experiences in school-based organized activities may also be influenced by their experiences in the broader school context. More research exploring intersectionality and other sources of discrimination and the various patterns of Latinx adolescents' experiences across other schoolbased settings are needed. Because adolescents' perceptions can vary, future work based on qualitative and pattern-centered approaches is needed to further understand the nuances of adolescents' experiences of discrimination in activities. Future research should consider the specific experiences of other minoritized racial/ethnic adolescents in order to identify profiles as well as differences across adolescents' experiences in order to inform more culturally responsive practices (Simpkins et al., 2017).

\section{Practical Implications}

The findings of this study have important implications for practice. First, for adolescents to reap the benefits of organized activities, they first have to participate. Therefore, it is crucial to create initiatives to better recruit and retain adolescents, particularly ethnic minority adolescents

\footnotetext{
${ }^{1}$ The study collected students' report on ethnic composition of the activity leaders (e.g., how many leaders in your activity are Latino? 0 (none), 1 (less than half), 2 (about half), 3 (more than half), 4 (all of them). Further analysis was run on whether leader's ethnic composition correlated with Latinx adolescents' experiences of ethnic discrimination and we failed to find any significance, $r=-.03, p=.69$. The leader ethnic composition was also dummy-coded in two ways: (a) 1 (more than half leaders Latino), 0 (less than half leaders Latino); (b) 1 (no Latino leaders), 0 (at least one Latino leader). Chi-square analyses were run on these two dummy variables with Latinx adolescents' peer ethnic discrimination profiles. No significance was revealed in the association of these variables and the ethnic discrimination profiles, Chi-square $(3)=.28, p=.96$; Chi-square (3) $=2.09, p=.55$, respectively.
} 


\section{Peer Ethnic Discrimination in Organized Activities}

or adolescents from groups who are the numeric minority in the activity. In fact, adolescents may not enroll if the activity has a reputation of being unwelcoming. Leaders might want to talk to adolescents who do not attend to see what they think of the activity and engage in efforts for other adolescents to see what it is like (e.g., initiatives where enrolled adolescents bring a friend). Once adolescents join, initiatives should be created to ensure that all groups, particularly ones who are the numeric minority, have a voice in activities and to improve peer relationships in these settings.

Scholars argue that it is helpful to incorporate three approaches in activities: race/ethnicspecific, multicultural, and universalistic (Okamoto et al., 2012; Simpkins et al., 2017). Race/ethnic-specific approaches where leaders support the development and needs of particular groups will help promote positive youth development, such as racial/ethnic identity. Multicultural aspects where adolescents learn about and celebrate various groups would help address some of the issues adolescents voiced about negative beliefs others held about their group. The universalistic approach emphasizes shared experiences and identity of the group and work on general positive youth development. In the case of peer relations, we believe it would be particularly helpful to build adolescents' social and emotional skills. Organized activities that have adopted social and emotional learning in their curriculum encourage adolescents to be accepting, sensitive, and welcoming to diverse experiences that lead to more positive relationships (Tiende \& Guskin, 2018). One specific practice that adults can implement to promote a more inclusive space for Latinx adolescents is through collaborative learning opportunities. Collaborative learning allows Latinx adolescents to demonstrate and leverage their individual strengths and cultural assets related to the importance of communal goals and interdependence in their communities (Yu et al., 2020). These opportunities allow adolescents to interact and get to know each other on a deeper level and see each other as sources of support which in turn may reduce discrimination among adolescents.

It is also vital for adults and activity leaders to intervene when observing instances of peer victimization including peer ethnic discrimination. The levels of perceived peer ethnic discrimination were fairly low among the adolescents in this study. This is likely, in part, because adolescents "vote with their feet" and probably leave an activity where they have faced substantial ethnic discrimination. Though the levels of peer ethnic discrimination were fairly low, they were associated with lower engagement and less positive experiences. Leaders will need to be alert and responsive to the various ways, including the not-so-obvious ways related to 


\section{Peer Ethnic Discrimination in Organized Activities}

negative beliefs and ethnic stereotypes, that peer ethnic discrimination can negatively affect adolescents' experiences in these settings (Simpkins et al., 2017).

When leaders have encountered culture-related incidents, ignoring the incident or engaging only in an adult-driven, limited discussion with adolescents was not the most helpful (Gutiérrez et al., 2017). Rather, the most helpful strategy was to have an immediate, constructive response where leaders actively listened and helped facilitate adolescents' discussion and reflective dialog. By implementing culturally responsive approaches rather than colorblind approaches, where race/ethnicity "does not matter" (Simpkins et al., 2017), these settings can provide an ideal context for promoting adolescents' awareness and understanding of their ethnic identity. In turn, promoting adolescents' ethnic identity may serve as a protective factor against the harmful effects of discrimination (Umaña-Taylor et al., 2015).

\section{Conclusion}

Our study has built on the strengths of prior research that qualitatively examined Latinx adolescents' experiences with discrimination within activities (e.g., Lin et al., 2016). These discriminatory experiences are not related only to ethnic composition in activities but also to whether adolescents participate and their experiences while participating. The pattern-centered approach further allowed us to give meaningful descriptions of the subtle, but critical variations in the peer ethnic discrimination adolescents experienced. Our findings are conducive to educators and organized activity leaders who hope to identify adolescents who may be most vulnerable to such discrimination and will help them to understand possible opportunities to support adolescents' adaptive functioning in response to these negative experiences.

\section{Acknowledgements}

This project was funded in part by the William T. Grant Young Scholars Award (\#7936) to Sandra D. Simpkins and a William T. Grant Award (\#181735) to Sandra D. Simpkins and Cecilia Menjívar.

\section{References}

Afterschool Alliance (2014). Taking a deeper dive into afterschool: Positive outcomes and promising practices. ERIC Clearinghouse. 
Journal of Youth Development | http://jyd.pitt.edu/ | Vol. 15 Issue 6 DOI 10.5195/jyd.2020.938 Peer Ethnic Discrimination in Organized Activities

Bejarano, C. L. (2005). ¿Qué onda? [What's up?] Urban youth cultures and border identity. The University of Arizona Press.

Bellmore, A., Nishina, A., You, J. I., \& Ma, T. L. (2012). School context protective factors against peer ethnic discrimination across the high school years. American Journal of Community Psychology, 49(1-2), 98-111. https://doi.org/10.1007/s10464-011-9443-0

Benner, A. D., \& Graham, S. (2011). Latino adolescents' experiences of discrimination across the first 2 years of high school: Correlates and influences on educational outcomes. Child development, $82(2), 508-519$.

Benner, A. D., Wang, Y., Shen, Y., Boyle, A. E., Polk, R., \& Cheng, Y. P. (2018). Racial/ethnic discrimination and well-being during adolescence: A meta-analytic review. American Psychologist, 73(7), 855-883. http://dx.doi.org/10.1037/amp0000204

Bronfenbrenner, U., \& Morris, P. A. (1998). The ecology of developmental processes. In W. Damon \& R. M. Lerner (Eds.), Handbook of child psychology: Theoretical models of human development (pp. 993-1028). John Wiley \& Sons.

Borden, L. M., Perkins, D. F., Villarruel, F. A., \& Stone, M. R. (2005). To participate or not to participate: That is the question. New Directions for Youth Development, 2005(105), 33-49.

Brown, C., \& Bigler, R. S. (2005). Children's perceptions of discrimination: A developmental model. Child Development, 76(3), 533-553. https://doi.org/10.1111/j.1467-8624.2005.00862.x

Byrd, C. M., \& Andrews, D. J. C. (2016). Variations in students' perceived reasons for, sources of, and forms of in-school discrimination: A latent class analysis. Journal of School Psychology, 57, 1-14. https://doi.org/10.1016/j.jsp.2016.05.001

Collins, L. M. and Lanza, S. T. (2010) Latent class and latent transition analysis: with applications in the social, behavioral and health sciences. Wiley.

Davalos, D. B., Chavez, E. L., \& Guardiola, R. J. (1999). The effects of extracurricular activity, ethnic identification, and perception of school on student dropout rates. Hispanic Journal of behavioral sciences, 21(1), 61-77.

Dawes, N. P., \& Larson, R. (2011). How youth get engaged: Grounded-theory research on motivational development in organized youth programs. Developmental Psychology, 4Х1), 259-269. http://dx.doi.org/10.1037/a0020729

DeGarmo, D. S., \& Martinez C. R., Jr. (2006). A culturally informed model of academic well-being for Latino youth: The importance of discriminatory experiences and social support. Family relations, 55(3), 267-278. https://doi.org/10.1111/j.1741-3729.2006.00401.x

Dworkin, J., \& Larson, R. (2007). Adolescents' negative experiences in organized youth activities. Journal of Youth Development, 1(3), 44-62. https://doi.org/10.5195/jyd.2007.373 
Journal of Youth Development | http://jyd.pitt.edu/ | Vol. 15 Issue 6 DOI 10.5195/jyd.2020.938

Peer Ethnic Discrimination in Organized Activities

Echols, L., \& Graham, S. (2018). Meeting in the Middle: The Role of Mutual Biracial Friends in Cross-Race Friendships. Child development, 91(2). 401-416. https://doi.org/10.1111/cdev.13179

Erbstein, N., \& Fabionar, J. O. (2019). Supporting Latinx Youth Participation in Out-of-School Time Programs: A Research Synthesis. Afterschool Matters, 29, 17-27.

Ettekal, A. V., Gaskin, E. R., Lin, A. R., \& Simpkins, S. D. (2015). "You gotta respect": Mexican-origin adolescents' perspectives on respect in organized activities. Journal of Youth Development, 10(3), 74-88.

Federal Interagency Forum on Child and Family Statistics. (2016). America's children in brief: Key national indicators of well-being, 2016. U.S. Government Printing Office.

Fisher, C. B., Wallace, S. A., \& Fenton, R. E. (2000). Discrimination distress during adolescence. Journal of Youth and Adolescence, 29(6), 679-695. https://doi.org/10.1023/A:1026455906512

Fredricks, J. A., \& Simpkins, S. D. (2013). Organized out-of-school activities and peer relationships: Theoretical perspectives and previous research. New Directions for Child and Adolescent Development, 2013(140), 1-17.

Garcia Coll, C. , Crnic, K., Lamberty, G., Wasik, B. H., Jenkins, R., Garcia, H. V., \& McAdoo, H. P. (1996). An integrative model for the study of developmental competencies in minority children. Child development, 675), 1891-1914.

Garnett, B. R., Masyn, K. E., Austin, S. B., Miller, M., Williams, D. R., \& Viswanath, K. (2014). The intersectionality of discrimination attributes and bullying among youth: An applied latent class analysis. Journal of Youth and Adolescence, 43(8), 1225-1239.

Gast, M. J., Okamoto, D. G., \& Feldman, V. (2017). "We only speak English here" English dominance in language diverse, immigrant after-school programs. Journal of Adolescent Research, 32(1), 94121. https://doi.org/10.1177/0743558416674562

Greene, M. L., Way, N., \& Pahl, K. (2006). Trajectories of perceived adult and peer discrimination among Black, Latino, and Asian American adolescents: Patterns and psychological correlates. Developmental Psychology, 42(2), 218-236. http://dx.doi.org/10.1037/0012-1649.42.2.218

Gutiérrez, V., Larson, R. W., Raffaelli, M., Fernandez, M., \& Guzman, S. (2017). How staff of youth programs respond to culture-related incidents: Nonengagement versus going "full-right-in". Journal of Adolescent Research, 32(1), 64-93.

Hurd, N., \& Deutsch, N. (2017). SEL-focused after-school programs. The Future of Children, 271), 95115.

Huynh, V. W., \& Fuligni, A. J. (2010). Discrimination hurts: The academic, psychological, and physical well-being of adolescents. Journal of Research on adolescence, 20(4), 916-941. 
Journal of Youth Development | http://jyd.pitt.edu/ | Vol. 15 Issue 6 DOI 10.5195/jyd.2020.938

Peer Ethnic Discrimination in Organized Activities

Johnston, K. E., \& Delgado, M. Y. (2004). Mexican American adolescents'experiences with ethnic discrimination. Poster session presented at the biennial meeting of the Society for Research on Adolescence, Baltimore, MD.

Knifsend, C. A., Camacho-Thompson, D. E., Juvonen, J., \& Graham, S. (2018). Friends in activities, school-related affect, and academic outcomes in diverse middle schools. Journal of Youth and Adolescence, 4オ6), 1208-1220.

Knight, G. P., Roosa, M. W., \& Umaña-Taylor, A. J. (2009). Studying ethnic minority and economically disadvantaged populations: Methodological challenges and best practices. American Psychological Association. https://doi.org/10.1037/11887-000

Lee, R. T., Perez, A. D., Boykin, C. M., Mendoza-Denton, R. (2019). On the prevalence of racial discrimination in the United States. PLOS ONE, 14(1): e0210698. https://doi.org/10.1371/journal.pone.0210698

Lerner, R. M., Wang, J., Hershberg, R. M., Buckingham, M. H., Harris, E. M., Tirrell, J. M., Bowers, E. P., \& Lerner, J. V. (2017). Positive youth development among minority youth: A relational developmental systems model. In Handbook on positive development of minority children and youth (pp. 5-17). Springer.

Lin, A. R., Menjívar, C., Vest Ettekal, A., Simpkins, S. D., Gaskin, E. R., \& Pesch, A. (2016). "They will post a law about playing soccer" and other ethnic/racial microaggressions in organized activities experienced by Mexican-origin families. Journal of Adolescent Research, 31(5), 557-581.

Lorenzo-Blanco, E. I., Unger, J. B., Oshri, A., Baezconde-Garbanati, L., \& Soto, D. (2016). Profiles of bullying victimization, discrimination, social support, and school safety: Links with Latino/a youth acculturation, gender, depressive symptoms, and cigarette use. American Journal of Orthopsychiatry, 86(1), 37-48. https://doi.org/10.1037/ort0000113

Mahoney, J. L., Vandell, D. L., Simpkins, S., \& Zarrett, N. (2009). Adolescent out-of-school activities. In R. M. Lerner \& L. Steinberg (Eds.), Handbook of adolescent psychology: Contextual influences on adolescent development (p. 228-269). John Wiley \& Sons. https://doi.org/10.1002/9780470479193.adlpsy002008

Moore, E. W. G., \& Hansen, D. (2012). Construct-validity of the engagement with challenge measure for adolescents: Structural- and criterion-validity evidence. Psychology, 3, 923-933. https://doi.org/10.4236/psych.2012.310139

National Center for Education Statistics, U.S. Department of Education. (2015). Federal programs for education and related activities. In The condition of education 2015 (chap. 2). https://nces.ed.gov/pubs2015/2015144.pdf 
Journal of Youth Development | http://jyd.pitt.edu/ | Vol. 15 Issue 6 DOI 10.5195/jyd.2020.938

Peer Ethnic Discrimination in Organized Activities

Ngo, B. (2017). Naming their world in a culturally responsive space: Experiences of Hmong adolescents in an after-school theatre program. Journal of Adolescent Research, 32(1), 37-

63. https://doi.org/10.1177/0743558416675233

Nishina, A., \& Bellmore, A. (2010). When might peer aggression, victimization, and conflict have its largest impact? Microcontextual considerations. The Journal of Early Adolescence, 30(1), 5-26. https://doi.org/10.1177/0272431609350928

Niwa, E. Y., Way, N., \& Hughes, D. L. (2014). Trajectories of ethnic-racial discrimination among ethnically diverse early adolescents: Associations with psychological and social adjustment. Child Development, 85(6), 2339-2354. https://doi.org/10.1111/cdev.12310

Nylund, K. L., Asparouhov, T., \& Muthén, B. O. (2007). Deciding on the number of classes in latent class analysis and growth mixture modeling: A Monte Carlo simulation study. Structural equation modeling: A multidisciplinary Journal, 14(4), 535-569.

Nylund, K., Bellmore, A., Nishina, A., \& Graham, S. (2007). Subtypes, severity, and structural stability of peer victimization: What does latent class analysis say? Child development, 78(6), 1706-1722. https://doi.org/10.1111/j.1467-8624.2007.01097.x

Okamoto, D., Gast, M., \& Feldman, V. (2012, August). Managing diversity in youth-serving organizations. Paper presented at the annual meeting of the American Sociological Association, Denver, CO.

Rosenthal, R., \& Vandell, D. L. (1996). Quality of care at school-aged child-care programs: Regulatable features, observed experiences, child perspectives, and parent perspectives. Child Development, 67, 2434-2445. https://doi.org/10.1111/j.1467-8624.1996.tb01866.x

Schaefer, D. R., Simpkins, S. D., Vest, A. E., \& Price, C. D. (2011). The Contribution of Extracurricular Activities to Adolescent Friendships: New Insights Through Social Network Analysis. Developmental Psychology, 4オ4), 1141-1152. https://doi.org/10.1037/a0024091

Seaton, E. K., \& Yip, T. (2009). School and neighborhood contexts, perceptions of racial discrimination, and psychological well-being among African American adolescents. Journal of youth and adolescence, 38(2), 153-163.

Shernoff, D. J., \& Vandell, D. L. (2007). Engagement in after-school program activities: Quality of experience from the perspective of participants. Journal of Youth and Adolescence, 36(7), 891903.

Simpkins, S. D., Delgado, M. Y., Price, C. D., Quach, A., \& Starbuck, E. (2013). Socioeconomic status, ethnicity, culture, and immigration: Examining the potential mechanisms underlying Mexicanorigin adolescents' organized activity participation. Developmental Psychology, 49(4), 706.

Simpkins, S. D., O'Donnell, M., Delgado, M. Y., \& Becnel, J. N. (2011). Latino adolescents' participation in extracurricular activities: How important are family resources and cultural orientation? Applied Developmental Science, 15(1), 37-50. https://doi.org/10.1080/10888691.2011.538618 
Journal of Youth Development | http://jyd.pitt.edu/ | Vol. 15 Issue 6 DOI 10.5195/jyd.2020.938 Peer Ethnic Discrimination in Organized Activities

Simpkins, S. D., Riggs, N. R., Ngo, B., Vest Ettekal, A., \& Okamoto, D. (2017). Designing culturally responsive organized after-school activities. Journal of Adolescent Research, 32(1), 11-36. https://doi.org/10.1177/0743558416666169

Smith, E. P., Osgood, D. W., Caldwell, L., Hynes, K., \& Perkins, D. F. (2013). Measuring collective efficacy among children in community-based afterschool programs: Exploring pathways toward prevention and positive youth development. American Journal of Community Psychology, 52(12), 27-40.

Tiende, L., \& Guskin, K. (2018). Deepening the teen outreach program (TOP) focus on social and emotional learning (SEL): A case study of curriculum and training revision and early impact. http://www.scefdn.org/what-we-do/social-emotional-learning/\#implementing-social-andemotional-learning-lessons-from-three-national-organizations

Umaña-Taylor, A. J., Tynes, B. M., Toomey, R. B., Williams, D. R., \& Mitchell, K. J. (2015). Latino adolescents' perceived discrimination in online and offline settings: An examination of cultural risk and protective factors. Developmental Psychology, 51(1), 87-100. https://doi.org/10.1037/a0038432

Williams, J. L., \& Deutsch, N. L. (2016). Beyond between-group differences: Considering race, ethnicity, and culture in research on positive youth development programs. Applied Developmental Science, 20(3), 203-213. https://doi.org/10.1080/10888691.2015.1113880

Yu, M. B., Liu, Y., Hsieh, T., Lee, G., Simpkins, S. D., \& Pantano, A. (2020). "Working together as a team really gets them fired up": Afterschool program mentoring strategies to promote collaborative learning among adolescent participants. Applied Developmental Science. 1-15. https://doi.org/10.1080/10888691.2020.1800467 